\title{
Representation of Goal and Movements without Overt Motor Behavior in the Human Motor Cortex: A Transcranial Magnetic Stimulation Study
}

\author{
Luigi Cattaneo, ${ }^{1}$ Fausto Caruana, ${ }^{2}$ Ahmad Jezzini, ${ }^{2}$ and Giacomo Rizzolatti ${ }^{2,3}$ \\ ${ }^{1}$ Centro Interdipartimentale Mente/Cervello, Università di Trento, 38100 Mattarello, Italy, and ${ }^{2}$ Dipartimento di Neuroscienze, Università di Parma, \\ and ${ }^{3}$ Istituto Italiano di Tecnologia, Unità di Parma, Parma 43100, Italy
}

\begin{abstract}
We recorded motor-evoked potentials (MEPs) to transcranial magnetic stimulation from the right opponens pollicis (OP) muscle while participants observed an experimenter operating two types of pliers: pliers opened by the extension of the fingers and closed by their flexion ("normal pliers") and pliers opened by the flexion of the fingers and closed by their extension ("reverse pliers"). In one experimental condition, the experimenter merely opened and closed the pliers; in the other, he grasped an object with them. In a further condition, the participants imagined themselves operating the normal and reverse pliers. During the observation of actions devoid of a goal, the MEP amplitudes, regardless of pliers used, reflected the muscular pattern involved in the execution of the observed action. In contrast, during the observation of goal-directed actions, the MEPs from OP were modulated by the action goal, increasing during goal achievement despite the opposite hand movements necessary to obtain it. During motor imagery, the MEPs recorded from OP reflected the muscular pattern required to perform the imagined action. We propose that covert activity in the human motor cortex may reflect different aspects of motor behavior. Imagining oneself performing tool actions or observing tool actions devoid of a goal activates the representation of the hand movements that correspond to the observed ones. In contrast, the observation of tool actions with a goal incorporates the distal part of the tool in the observer's body schema, resulting in a higher-order representation of the meaning of the motor act.
\end{abstract}

\section{Introduction}

The main function of the motor areas of the frontal cortex is that of generating voluntary movements. However, motor areas are also active in the absence of overt motion, as during motor imagery (Jeannerod, 2001) or during observation of movements done by others (Rizzolatti and Craighero, 2004; Cattaneo and Rizzolatti, 2009). The activity of motor areas during motor imagery is thought to reflect a preparation to move not followed by an overt motor behavior. This activity is, therefore, still related, albeit in a particular way, to movement generation (Jeannerod, 2001).

This does not seem to be the case for the activation of motor areas during the observation of goal-directed motor acts done by others. On the basis of single-neuron experiments in non-human primates, this externally determined activation is considered to be functional to the understanding of the goal of the observed motor acts (Umiltà et al., 2001; Kohler et al., 2002). Notwithstanding this largely accepted view, transcranial magnetic stimulation (TMS) experiments in humans typically fail to show action-related modulations in the observer's motor cortex, instead revealing a faithful replica of the observed movements (Fadiga et al., 1995; Strafella and Paus, 2000; Gangitano et

Received June 4, 2009; revised July 28, 2009; accepted July 29, 2009.

This study was supported by European Union (EU) Contract 012738 , Neurocom, and by Programmi di ricerca di Rilevante Interesse Nazionale 2006 to G.R. F.C. and A.J. were supported by EU Contract 027017, Neuroprobes.

Correspondence should be addressed to Dr. Luigi Cattaneo, Centro Interdisciplinare Mente/Cervello, Università di Trento, Via delle Regole, 101, 38100 Mattarello, Italy. E-mail: luigi.cattaneo@unitn.it.

D01:10.1523/JNEUROSCI.2605-09.2009

Copyright $\odot 2009$ Society for Neuroscience ～0270-6474/09/2911134-05\$15.00/0 al., 2001; Aziz-Zadeh et al., 2002; Maeda et al., 2002; Borroni et al., 2005; Urgesi et al., 2006). This appears to suggest that, in humans, the exogenous activation of the motor cortex by observation of actions of others may share the same basic mechanisms (motor preparation) as its endogenous activation during motor imagery (Clark et al., 2004), and more broadly, it seemingly contradicts a role of cortical motor areas in goal coding.

In the present study, we investigated the covert activation of the motor cortex (observation and imagery) by using a paradigm in which we dissociated action goals from movements to achieve them by using two types of tools: normal pliers and reverse pliers. With these two types of pliers, the same goal (grasping an object) is obtained by performing opposite movements: with normal pliers, the grasping is achieved by means of the flexion of the fingers; whereas with the reverse pliers, it is achieved by means of the extension of the fingers (see Materials and Methods). Normal and reverse pliers were used to grasp objects ("goal" condition) and to perform the same opening-closing movements but without a target ("no-goal" condition). In experiment 1 (observation), participants observed an experimenter operating pliers; whereas in experiment 2 (motor imagery), they imagined themselves operating them. In experiment 3 (observation after practice), participants observed an experimenter using the pliers (as in experiment 1) after they underwent a motor training with both tools. In all experiments, the EMG of the participants' right opponens pollicis (OP), a muscle that flexes the thumb, was recorded.

The aim of experiments 1 and 2 was to assess whether the covert motor representation recruited during the two tasks (ob- 
normal pliers
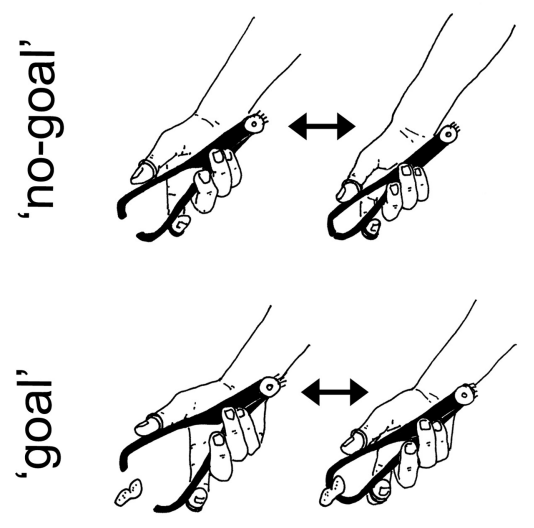

Figure 1. Diagram of experimental conditions in experiments 1,2, and 3. Two tools (normal and reverse pliers) were used in two action types (no-goal and goal). Normal pliers (left) are opened by extension of the thumb and index fingers and closed by their flexion. Reverse pliers (right) are opened by flexion of the thumb and index fingers and closed by their extension. In the no-goal condition (top), tools were first opened and then closed repetitively without any object to grasp. In the goal condition (bottom), the same movements were performed to grasp small objects. Magnetic stimuli were delivered for each condition just before the moment of maximal aperture and maximal closure of the tool's arms, as signaled by built-in potentiometers. The tasks were to observe the actions in experiment 1 and in experiment 3 and to imagine doing the actions in experiment 2.

servation and motor imagery) is the same as the one recruited during movement preparation or whether it reflects cognitive functions (representation of the goal of the action). Experiment 3 was a control experiment to assess whether the effects of experiment 1, especially during the observation of the reverse pliers, could be attributable to the lack of experience of the volunteers with that tool.

\section{Materials and Methods}

\section{Experiment 1 (action observation)}

\section{Subjects and experimental protocol}

Fourteen volunteers, aged between 24 and 36 years, took part in the experiment. All were right handed and free of any history of neurological disorders. Written informed consent was obtained from all participants before entering the study, which was previously approved by the local Ethical Committee. All participants were naive to the aim of the study. In each experimental session, participants were comfortably seated in a chair with a fixed headrest and instructed to watch carefully the actions done by an experimenter standing in front of them. As a control for attention, the participants were told that they would have been debriefed about what they had seen after the end of the experiment. The experimenters operated the pliers with their right hand and performed two different types of actions with both the normal and reverse pliers: (1) goal-directed actions, i.e., grasping a peanut with the pliers and dropping it, and (2) no-goal actions, i.e., rhythmically opening and closing the pliers, not directed to any object. The experimenter took care to operate the pliers with the same pace and with same amount of proximal movements in both goal and no-goal conditions.

In each trial, the experimenter repeated the movement of opening and closing the pliers for at least six cycles. Participants received single TMS pulses over the hand motor cortex of the left hemisphere, synchronized with the opening or the closing phases of the pliers. Magnetic pulses were randomly delivered between the second and the fifth movement repetitions. The four different actions were repeated in a random order. Therefore, a total of eight conditions were presented in a $2 \times 2 \times 2$ design: 2 pliers (normal or reverse) $\times 2$ actions (goal condition or no-goal condition) $\times 2$ phases (opening or closing). Each condition was repeated 10 times, for a total of 80 stimuli.

\section{Pliers}

Two types of tools were used: normal and reverse pliers. Normal pliers (Fig. 1, left) are opened by extension of the thumb and index fingers and closed by their flexion. Reverse pliers (Fig. 1, right) are opened by flexion of the thumb and index fingers and closed by their extension. The pliers were passive, i.e., did not contain a spring, and therefore, to be operated, they required an active contraction of alternatively the extensor or of the flexor muscles. The OP muscle was, therefore, active in flexing the thumb during pliers' closure with normal pliers and during pliers opening with reverse pliers. Both pliers were used in two action types: no-goal and goal. In the no-goal condition (Fig. 1, top line), tools were first opened and then closed repetitively without any object to grasp. In the goal condition (Fig. 1, bottom line), the same movements were performed to grasp small objects. Both tools had a built-in potentiometer (connected to a $4.5 \mathrm{~V}$ battery) that indicated the angle formed by the two arms of the pliers.

\section{Stimulation and recording}

TMS was delivered randomly from the second to the fifth cycle of the pliers' movements. This procedure was adopted to avoid that the participants could predict the occurrence of the stimuli. On the other hand, the stimuli were delivered at a time when the action had already been seen at least once. Synchronization between the pliers' movements and TMS was achieved with the built-in potentiometer. The signal from the potentiometers triggered the TMS, which was delivered few milliseconds before maximal aperture or maximal closure (see supplemental Fig. 1, available at www.jneurosci.org as supplemental material).

TMS was applied using a Magstim 200 stimulator connected to a $7 \mathrm{~cm}$ figure-of-eight coil. The coil was applied tangentially to the scalp with the handle pointing backwards and laterally with a $45^{\circ}$ angle to the midline. The stimulus intensity was set to obtain motor-evoked potentials (MEPs) at rest with average amplitude of $1 \mathrm{mV}$. Recordings were made from the opponens pollicis muscle of the right hand with a couple of surface $\mathrm{Ag}-\mathrm{AgCl}$ electrodes. The signal was amplified $1000 \times$ by means of a 1902 amplifier (Cambridge Electronic Design), sampled at $4 \mathrm{kHz}$, and stored for off-line analysis. Also, the signal from the pliers' potentiometers was sampled at $100 \mathrm{~Hz}$ and stored for subsequent analyses. Digital conversion and timing of the TMS pulses were performed with a micro $1401 \mathrm{mk} 2$ unit (Cambridge Electronic Design) controlled by the Spike2 software (Cambridge Electronic Design).

\section{Data analysis}

Digital bandpass filtering of $10 \mathrm{~Hz}-2 \mathrm{kHz}$ was applied, and peak-topeak amplitude of single MEPs was calculated and averaged within conditions. Sweeps showing muscular activity $>50$ microvolt in the $500 \mathrm{~ms}$ preceding the stimulus were discarded. Statistical analysis was performed on mean MEP amplitudes as dependent variable in an ANOVA with three within-subjects factors, each with two levels: pliers type (normal or reverse), action type (goal or no-goal) and movement phases of the fingers (flexion or extension). Post hoc analyses were made with Newman-Keuls test.

To check for accuracy of timing, the potentiometers' signal was averaged offline by aligning it to the TMS pulses within each condition (supplemental Fig. 1, available at www.jneurosci.org as supplemental material).

\section{Experiment 2 (motor imagery)}

Subjects and experimental protocol

Twelve right-handed volunteers, aged between 24 and 36 years, took part in the experiment. For participant criteria selection and other details, see Experiment 1, above.

In each experimental session, subjects were comfortably seated in a chair with a fixed headrest. They were asked to imagine themselves per- 
forming the two actions of experiment 1 using either normal or reverse pliers. Every trial began with a vocal instruction, on which of the four actions (i.e., goal-directed or no-goal actions either with normal or reverse pliers) has to be imagined. Because most participants found some difficulties to imagine themselves operating the reverse pliers, they were trained to operate both the reverse and normal pliers for a few hours before the experiment. They were then required to demonstrate their skill grasping small objects.

A series of two alternating tones, rhythmically delivered at a frequency of $0.7 \mathrm{~Hz}$ by a personal computer, guided the participants in timing the imagined movements. They were instructed to pace the imagined movement so that maximal opening and maximal closure of the pliers coincided with the two tones, respectively. It must be noted that the timing instructions concerned the opening and closure of the pliers' tips and not of the hand.

The order of trials was randomized. Subjects received single TMS pulses over the hand motor cortex of the left hemisphere. TMS was delivered in correspondence with the pacing tones, i.e., either during the imagined maximal aperture or closure of the pliers. Analogously to experiment 1 , the TMS pulse was delivered randomly from the second and the fifth cycle of the movements, to make its occurrence unpredictable to the participant. A total of eight conditions was presented in a $2 \times 2 \times 2$ design: 2 pliers (normal or reverse) $\times 2$ actions (goal condition or no-goal condition) $\times 2$ phases (opening or closing). Each condition was repeated for 10 times. As a control, participants were required to report in every trial, immediately after the TMS pulse, at what point of the imagined action their action was at the moment of TMS.

Stimulation, recordings, and data analysis TMS and recordings form the opponens pollicis muscle parameters were the same as in experiment 1 , and data processing was performed similarly. Special care was taken to discard all trials in which muscle activity $>50$ microvolt appeared in the $500 \mathrm{~ms}$ preceding the stimulus. Statistical analysis was similarly performed on mean MEP amplitudes as dependent variable in an ANOVA with three within-subjects factors, each with two levels: pliers type (normal or reverse), action type (goal or no-goal) and movement phases of the fingers (flexion or extension). Post hoc analyses were made with Newman-Keuls test.

\section{Experiment 3 (action observation after practice) \\ Pliers}

The pliers used in experiment 3 were the same used in experiment 1.

\section{Subjects and experimental protocol}

Eight right-handed volunteers, aged between 23 and 30 years, took part in the experiment. For participant criteria selection and other details, see Experiment 1 , above. For the $24 \mathrm{~h}$ before the TMS session, participants were asked to train themselves extensively to use the reverse pliers in their daily activities. After the training, they were required to demonstrate their expertise by accurately grasping and placing small objects with that tool. In this experiment, subjects watched only actions made with reverse pliers. are given.

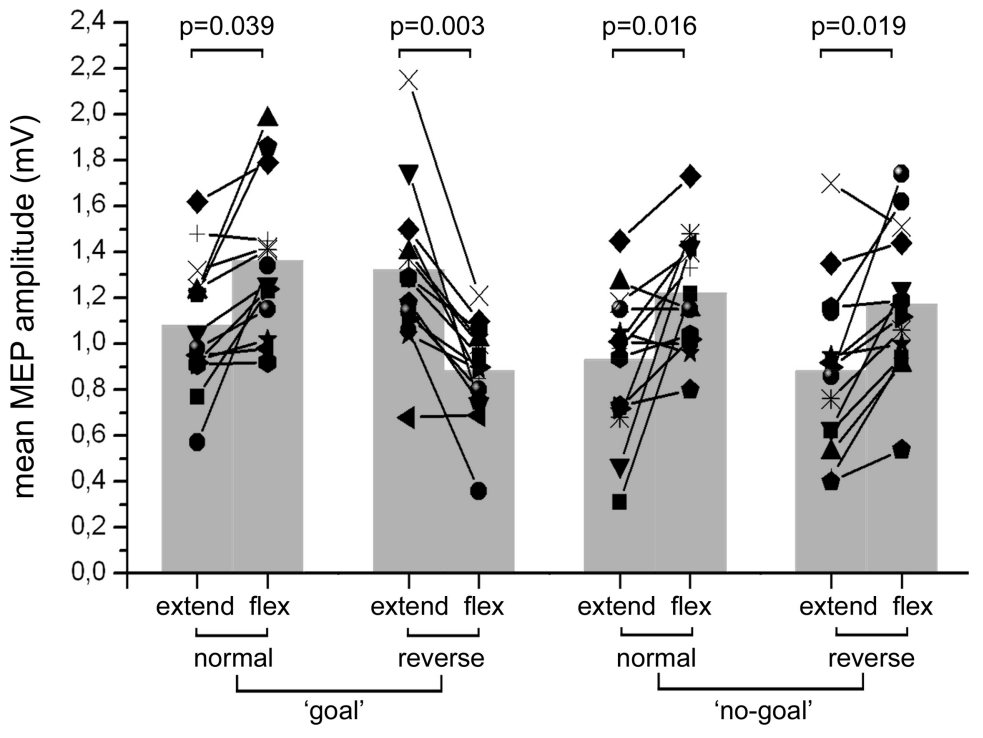

Figure 2. Modulation of MEPs during action observation. Mean MEP amplitudes recorded from the right opponens pollicis uring the observation of the experimenter operating the normal and reverse tools, in both the goal and no-goal conditions. ine-symbol, whereas mean values of the group are represented as gray columns. $p$ values from the relevant post hoc comparisons

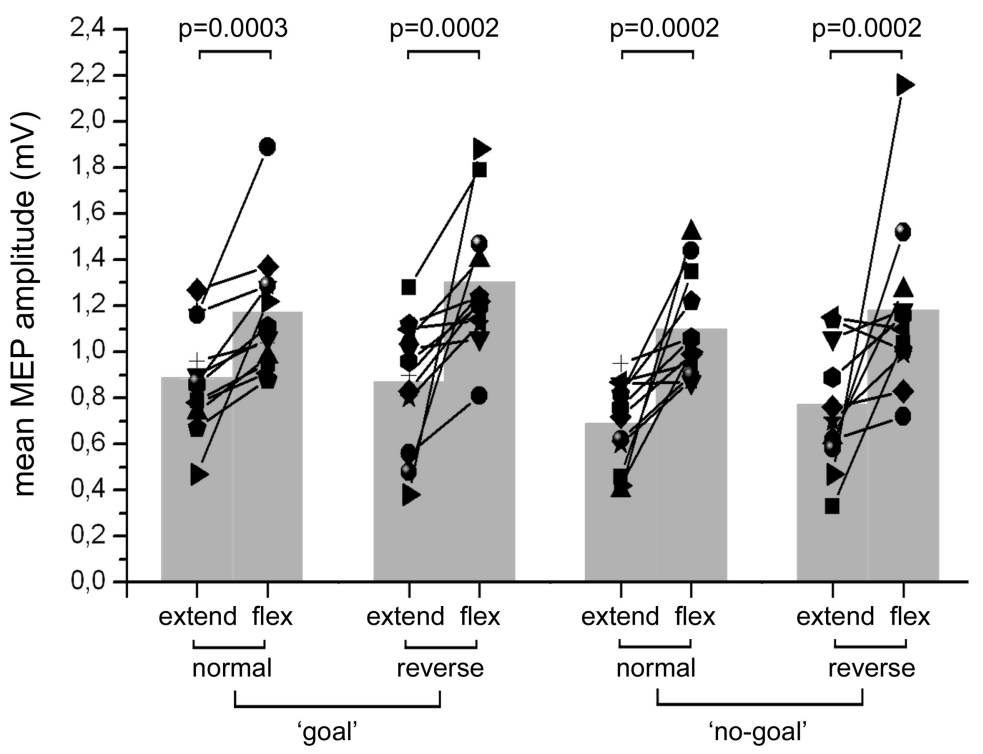

Figure 3. Modulation of MEPs during motor imagery. Mean MEP amplitudes recorded from the right opponens pollicis during the imagination of motor behaviors using the normal and reverse tools in both the goal and no-goal conditions. "Extend" and "flex" refer to the imagined movements of the participants' thumb. Values from individual participants are represented as line-symbol, whereas mean values of the group are represented as gray columns. $p$ values from the relevant post hoc comparisons are given.

Stimulation, recordings, and data analysis

The same modalities of stimulation and the same data analysis as in experiment 1 were applied.

\section{Results}

\section{Experiment 1 (action observation)}

Only $3 \%$ of trials were discarded because of electromyographic activity before the TMS. The analysis of the potentiometer signal in the four different conditions (goal or no-goal, normal or reverse pliers) showed an extremely coherent kinematics between conditions, with an intrinsic frequency of $0.7 \mathrm{~Hz}$ (see supplemental Fig. 1, available at www.jneurosci.org as supplemental material). 
The ANOVA showed a significant effect of the "movement phase" (fingers' extension or flexion) factor $\left(F_{(1,13)}=9.1512, p=\right.$ $0.00976)$, a significant interaction of "action type" (goal or no-goal) by phase $\left(F_{(1,13)}=17.095, p=0.00117\right)$ and of "pliers type" ("normal" and "reverse") by movement phase $\left(F_{(1,13)}=23.250, p=\right.$ $0.00033)$. The most relevant result was a significant interaction of the three factors: pliers type, action type, and movement phase $\left(F_{(1,13)}=17.666, p=0.00103\right)$. Post hoc comparisons showed a significant difference between MEP amplitudes in the opening and closing phases in all conditions. Mean values, however, were larger in the finger flexion phase than in the finger extension phase for the normal pliers both in the goal and no-goal condition and for the reverse pliers in the no-goal condition. Most interestingly, with reverse pliers in the goal condition, the MEP amplitude was larger in the finger extension phase than in the flexion phase. Individual results, mean values, and $p$ values of post hoc comparisons are shown in Figure 2.

\section{Experiment 2 (motor imagery)}

Four percent of trials were discarded because of muscular activity before TMS. The ANOVA showed a main effect of the factors action type $\left(F_{(1,11)}=6.8948, p=0.02358\right)$, with MEPs being larger when subjects imagined the goal-directed acts (mean, 1.06 $\mathrm{mV}$ ) than when they imagined the no-goal acts (mean, $0.92 \mathrm{mV}$ ), and movement phase $\left(F_{(1,11)}=18.131, p=0.00135\right)$, with MEPs larger when imagining the finger flexion (mean, $1.18 \mathrm{mV}$ ) rather than the finger extension (mean, $0.80 \mathrm{mV}$ ). Also comparisons between mean MEP amplitude in the flexion and extension phases were significant for all four conditions as shown in Figure 3 , where, also, individual values are plotted.

\section{Experiment 3 (action observation after practice)}

Two percent of trials were discarded because of electromyographic activity preceding the stimulus. The ANOVA showed a significant interaction of the two factors action type and movement phase $\left(F_{(1,7)}=20.893, p=0.003\right)$. As shown in Figure 4, post hoc analyses showed a significant difference between the finger extension and finger flexion phases in both action types, thus confirming the results of experiment 1 .

\section{Discussion}

There are two conditions in which the human motor cortex is active in the absence of overt movements: motor imagery and the observation of motor acts done by others. In the present study, we attempted to assess which aspects of motor behavior does the motor cortex code in these two conditions, namely, the movements necessary for achieving a goal or the goal of a given motor act regardless of the movements necessary to achieve it. To dissociate movements and goals, we used two tools that require an opposite set of movements to reach the same goal.

The first main result of our experiment was the demonstration that there is a clear difference between the cortical processing underlying motor imagery and that underlying motor act observation. In the first case, regardless of whether individuals imagine themselves executing purposeless movements or performing movements leading to a goal, the pattern of motor cortex activation is the same as the one that occurs when that individual performs overtly the imagined movements. The motor cortex excitability in motor imagery does not appear to be influenced by the presence of a goal in the task. In accord with previous interpretations, motor imagery appears to be essentially a motor preparation, i.e., a preparation to act not followed by an overt motor behavior (Jeannerod, 2001).

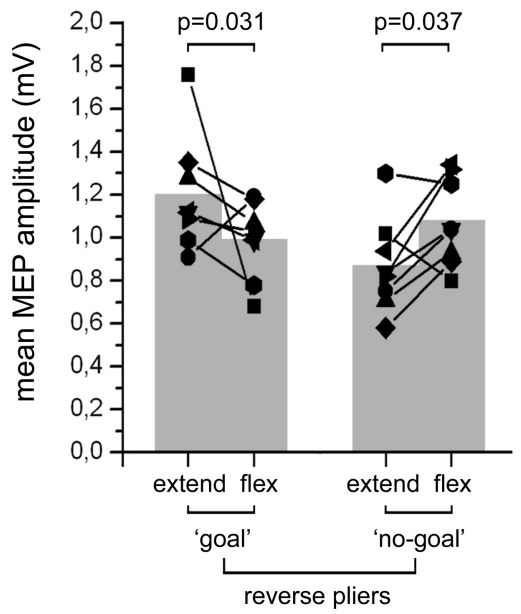

Figure 4. Modulation of MEPs during action observation after motor training. Mean MEP amplitudes recorded from the right opponens pollicis during observation of the extension and flexion phases of the experimenter's fingers, with the reverse pliers, in both the goal and nogoal conditions. Values from individual participants are represented as line-symbol, whereas mean values of the group are represented as gray columns. $p$ values from the relevant post hoc comparisons are given.

The activation picture in the case of observation of actions done by others is markedly different. When there is no goal in the observed behavior, the observer's motor cortex excitability reflects the movements performed by the agent. This was observed in the case of grasping done with normal pliers, where the cortical excitability of OP muscle increased during pliers closure, as well as in the case of reverse pliers, where the OP muscle cortical excitability increased during pliers aperture. However, when a goal is present in the observed motor behavior, the excitability of the motor cortex does not reflect any more the movements that the agent is doing but the movements necessary to reach the goal by the distal effector. Thus, during the observation of grasping with the reverse pliers, the OP muscle's cortical excitability increased, not during the thumb flexion but, paradoxically, during thumb extension.

What is the reason of this behavior? It is obvious that the hypothesis of motor preparation does not hold here. Rather, in accord with data obtained in monkeys (see below), it appears that when a goal is present in the observed motor behavior, the motor cortex codes the ultimate effect of the observed movements over the object (in our case grasping), regardless of what body parts are actually displaced to achieve it. A parallel to the goal-coding behavior described here during action observation is to be found in a recent neurophysiological study on monkeys where a tool-use paradigm as that of the present experiment was used (Umiltà et al., 2008). This study showed that "grasping" neurons in the ventral premotor cortex (area F5) controlling the closure of the hand were excited during the observation of grasping done with hands, with normal pliers as well as with reverse pliers. This happened despite the fact that in the last case the hand movement producing the closure of the pliers was the extension of the fingers rather than its flexion.

Importantly, the study of Umiltà et al. (2008) also showed that during active movements, most neurons in the ventral premotor cortex and many in the primary motor cortex discharged in relation to the goal of the movement. These neurons fired during closure of the pliers, when the monkeys used the normal pliers, and during their aperture, when the monkeys used the reverse pliers. This finding indicates that the same neurons control alter- 
natively flexors or extensor muscles in accordance to what set of muscles is needed to use to achieve the goal. Other studies also reported goal movement coding in the monkey cortical motor system (Rizzolatti et al., 1988; Alexander and Crutcher, 1990a,b; Kakei et al., 1999, 2001). In particular, Kakei et al. (1999, 2001) demonstrated that most neurons of the ventral premotor cortex and part of neurons of the primary motor cortex are modulated by high-order motor parameters such as the hand path necessary to reach a certain target in space, regardless of the muscle activation pattern required to reach it. The demonstration that during overt motor behavior the motor system is sensitive to extrinsic parameters such as target direction and action goal, indicates that a high-level representation of the action, filtered from intrinsic parameters, is present in the primary and premotor areas.

These neurophysiological data allow accounting for our results obtained during action observation. The observation of a visual pattern indicating a goal-directed motor act, like grasping, determines the activation of a grasping motor pattern both when the observed movement is the hand closure, as in the case of grasping with the hand, or the hand opening, as in the case of the reverse pliers. By virtue of this mechanism, the observer maps different types of actions but with the same goal on the same cortical motor neurons, translating the many possible motor behaviors that result in a grasp into one single feature of the observer's motor repertoire, i.e., a real grasp. In this way, the observer may generalize with his/her motor cortex the goal of an observed motor act regardless of the type of movement actually used to achieve it.

It is well established that the motor resonance to observed motor acts is strongly influenced by the motor familiarity of the observer with the observed acts (Calvo-Merino et al., 2005, 2006; Cross et al., 2006). In particular, this has been also shown for skilled actions made with tools such as chopsticks (Järveläinen et al., 2004). It could be, therefore, speculated that the "paradoxical" activation seen in the participants' motor system during observation of inverse pliers use can be attributable to their motor inexperience with the tool. The data from experiment 3 allowed us to rule out this possibility. They showed that the same pattern of activation is present in participants after extensive motor training.

In conclusion, the present data show that when individuals observe the behavior of another person, their motor cortical areas respond both to the goal of the observed actions and the movements necessary to achieve it. This dual information allows, through the motor resonance mechanism, the comprehension of others' actions based on both these features, their respective role varying according to which of them is more informative for understanding others' behavior.

\section{References}

Alexander GE, Crutcher MD (1990a) Neural representations of the target (goal) of visually guided arm movements in three motor areas of the monkey. J Neurophysiol 64:164-178.

Alexander GE, Crutcher MD (1990b) Preparation for movement: neural representations of intended direction in three motor areas of the monkey. J Neurophysiol 64:133-150.

Aziz-Zadeh L, Maeda F, Zaidel E, Mazziotta J, Iacoboni M (2002) Lateralization in motor facilitation during action observation: a TMS study. Exp Brain Res 144:127-131.

Borroni P, Montagna M, Cerri G, Baldissera F (2005) Cyclic time course of motor excitability modulation during the observation of a cyclic hand movement. Brain Res 1065:115-124.

Calvo-Merino B, Glaser DE, Grèzes J, Passingham RE, Haggard P (2005) Action observation and acquired motor skills: an FMRI study with expert dancers. Cereb Cortex 15:1243-1249.

Calvo-Merino B, Grèzes J, Glaser DE, Passingham RE, Haggard P (2006) Seeing or doing? Influence of visual and motor familiarity in action observation. Curr Biol 16:1905-1910.

Cattaneo L, Rizzolatti G (2009) The mirror neuron system. Arch Neurol 66:557-560.

Clark S, Tremblay F, Ste-Marie D (2004) Differential modulation of corticospinal excitability during observation, mental imagery and imitation of hand actions. Neuropsychologia 42:105-112.

Cross ES, Hamilton AF, Grafton ST (2006) Building a motor simulation de novo: observation of dance by dancers. Neuroimage 31:1257-1267.

Fadiga L, Fogassi L, Pavesi G, Rizzolatti G (1995) Motor facilitation during action observation: a magnetic stimulation study. J Neurophysiol 73:2608-2611.

Gangitano M, Mottaghy FM, Pascual-Leone A (2001) Phase-specific modulation of cortical motor output during movement observation. Neuroreport 12:1489-1492.

Järveläinen J, Schürmann M, Hari R (2004) Activation of the human primary motor cortex during observation of tool use. Neuroimage 23:187-192.

Jeannerod M (2001) Neural simulation of action: a unifying mechanism for motor cognition. Neuroimage 14:S103-S109.

Kakei S, Hoffman DS, Strick PL (1999) Muscle and movement representations in the primary motor cortex. Science 285:2136-2139.

Kakei S, Hoffman DS, Strick PL (2001) Direction of action is represented in the ventral premotor cortex. Nat Neurosci 4:1020-1025.

Kohler E, Keysers C, Umiltà MA, Fogassi L, Gallese V, Rizzolatti G (2002) Hearing sounds, understanding actions: action representation in mirror neurons. Science 297:846-848.

Maeda F, Kleiner-Fisman G, Pascual-Leone A (2002) Motor facilitation while observing hand actions: specificity of the effect and role of observer's orientation. J Neurophysiol 87:1329-1335.

Rizzolatti G, Craighero L (2004) The mirror-neuron system. Annu Rev Neurosci 27:169-192.

Rizzolatti G, Camarda R, Fogassi L, Gentilucci M, Luppino G, Matelli M (1988) Functional organization of inferior area 6 in the macaque monkey: II. Area F5 and the control of distal movements. Exp Brain Res 71:491-507.

Strafella AP, Paus T (2000) Modulation of cortical excitability during action observation: a transcranial magnetic stimulation study. Neuroreport 11:2289-2292.

Umiltà MA, Kohler E, Gallese V, Fogassi L, Fadiga L, Keysers C, Rizzolatti G (2001) I know what you are doing. A neurophysiological study. Neuron 31:155-165.

Umiltà MA, Escola L, Intskirveli I, Grammont F, Rochat M, Caruana F, Jezzini A, Gallese V, Rizzolatti G (2008) When pliers become fingers in the monkey motor system. Proc Natl Acad Sci U S A 105:2209-2213.

Urgesi C, Candidi M, Fabbro F, Romani M, Aglioti SM (2006) Motor facilitation during action observation: topographic mapping of the target muscle and influence of the onlooker's posture. Eur J Neurosci 23:25222530. 